\title{
Detection of absolute rotation using superfluid ${ }^{4} \mathrm{He}$
}

\author{
K. Schwab \\ California Institute of Technology, Pasadena CA 91125, USA \\ N. Bruckner and R. Packard \\ Physics Department, University of California, Berkeley CA 94720, USA \\ E-mail: packard@socrates.berkeley.edu
}

\begin{abstract}
We have developed the superfluid analog of the superconducting rf SQUID. Such a device is a quantum mechanically based, absolute gyroscope and has been used to sense the rotation of the Earth. Our device is fabricated using silicon processing techniques and forms a planer sensing loop of superfluid helium which couples to the applied rotation. A much more sensitive superfluid gyroscope based on the principals demonstrated with this device, might ultimately be used to detect the precession of our local inertial frame with respect to the fixed stars by the gravitomagnetic field of the rotating Earth. We compare the superfluid gyroscope against two other experiments aimed at detecting this general relativistic effect.
\end{abstract}

PACS: $67.40 . \mathrm{Vs}, 47.15 . \mathrm{Ki}, 67.40 . \mathrm{Hf}$

\section{Introduction}

It has long been appreciated that superfluid ${ }^{4} \mathrm{He}$ could be used as a reference to an absolute inertial frame [1-3]. In this paper we outline how we exploit the quantum properties of superfluid ${ }^{4} \mathrm{He}$ to construct an absolute gyroscope, discuss the recent success to observe the rotation of the Earth using a device which behaves similarly to an rf SQUID [4-6], and discuss the possibility of using a much improved superfluid gyroscope to observe the Lense-Thirring Effect of the rotating Earth.

\section{Circulation quantization in superfluids}

Here we show that if a Schrodinger-like equation of motion is assumed for the macroscopic order parameter of a superfluid, one concludes that circulation is quantized in units of $\kappa_{0}$ in the local inertial frame, where $\kappa_{0}=h / m_{4}$ and $m_{4}$ is the bare mass of the helium atom. Anandan [7] gives an elementary argument where one begins with the Gross-Pitaevsky equation for the superfluid order parameter $\Psi(\mathbf{r}, t)$ :

$$
i \hbar \frac{\partial \Psi}{\partial t}=-\frac{\hbar^{2}}{2 m_{4}} \nabla^{2} \Psi+V \Psi+g|\Psi|^{2} \Psi
$$

where $g$ is the strength of the self interactions of the superfluid and $V$ is from external potentials. We assume this to be true in the local inertial frame. In the usual way, if we evaluate the time derivative of this equation and of the complex conjugate equation, we can identify a continuity equation and a mass current density operator:

$$
\frac{\partial}{\partial t}\left(\Psi^{*} \Psi\right)+\nabla \cdot\left[\frac{\hbar}{2 i m_{4}}\left(\Psi^{*} \nabla \Psi-\Psi \nabla \Psi^{*}\right)\right]=0
$$

where we interpret the term on the left hand side to be the time derivative of the superfluid density, $\Psi^{*} \Psi$, and the next term to the divergence of the superfluid mass current. If we assume a stationary wave function of the form $\Psi(\mathbf{r}, t)=\Psi_{0}(\mathbf{r}) \exp (-i E t / \hbar)$, where $E$ is a constant and $\Psi_{0}=\sqrt{\rho} \exp (i \varphi)$, then the current operator becomes

$$
\mathbf{j}=\frac{\hbar}{2 i m_{4}}\left(\Psi^{*} \nabla \Psi-\Psi \nabla \Psi^{*}\right)=\rho \frac{\hbar}{m_{4}} \nabla \varphi .
$$

Thus the superfluid velocity is $v_{s}=\left(\hbar / m_{4}\right) \nabla \varphi$. If we evaluate the path integral of the velocity around a closed contour, we find

$$
\oint \mathbf{v} \cdot \mathbf{d} \mathbf{l}=\frac{\hbar}{m_{4}} \oint \nabla \varphi \cdot \mathbf{d} \mathbf{l}=\frac{\hbar}{m_{4}} 2 \pi n=n \kappa_{0},
$$

that circulation is quantized in units of $\kappa_{0}$. This is required if the complex order parameter $\Psi$ is to be 
single valued as we traverse around the path of integration.

Following from fundamental quantum mechanical assumptions, that is, the existence of a macroscopic order parameter and a Schrodinger-like equation of motion, it is found that circulation in a superfluid is quantized, and this quantization is with respect to a local inertial frame. By measuring these circulation states, we can use the superfluid as a reference to an absolute, non-rotating frame.

\section{Superfluid gyroscope}

An annular container with a septum and an aperture provide a means to exploit the quantization of circulation to measure rotation of the container with respect to the local inertial frame. If this container is rotating with angular velocity $\omega$, one finds that the superfluid will undergo essentially solid body motion along a contour between the inner and outer radius of the annulus [6]. In order that the circulation is to be quantized along a path threading the annulus and the aperture, a back-flow through the aperture is required:

$$
v_{a}=n \frac{\kappa_{0}}{l_{a}}-\omega R \frac{2 \pi R_{a}}{l_{a}},
$$

where $n$ is an integer, $l_{a}$ is the hydrodynamic length of the aperture, and $R_{a}$ is the radius of the annulus.

In our device, this back-flow is detected by measuring the apparent change in the onset of vortex nucleation in the aperture. These vortices nucleate at a superfluid velocity $v_{c} \pm \Delta v_{c}$, where $v_{c}$ is relative to the walls of the aperture, and $\Delta v_{c}$ is the spread in velocity from the stochastic (thermal or quantum) nucleation mechanisms. One can see that the superfluid gives a reference to an absolute, non-rotating, inertial frame, since the apparent change in the apparent critical velocity in the aperture will be zero in this frame.

Free vortices which are inevitably created when the superfluid sample cools through $T_{\lambda}$, will produce a non-zero bias through the aperture when the gyroscope is at rest. By making measurements at two opposing orientations of the sensing loop, one can reject this bias and detect only the signal from absolute rotation. This is the same technique used to make an absolute magnetometer using a superconducting rf SQUID [8].

We have demonstrated a device which is very similar in dynamics and operation to a superconducting rf SQUID. The device is a two hole, diaphragm-aperture superfluid oscillator and is fabricated from a $1.5 \mathrm{~cm}$ silicon square. It has a

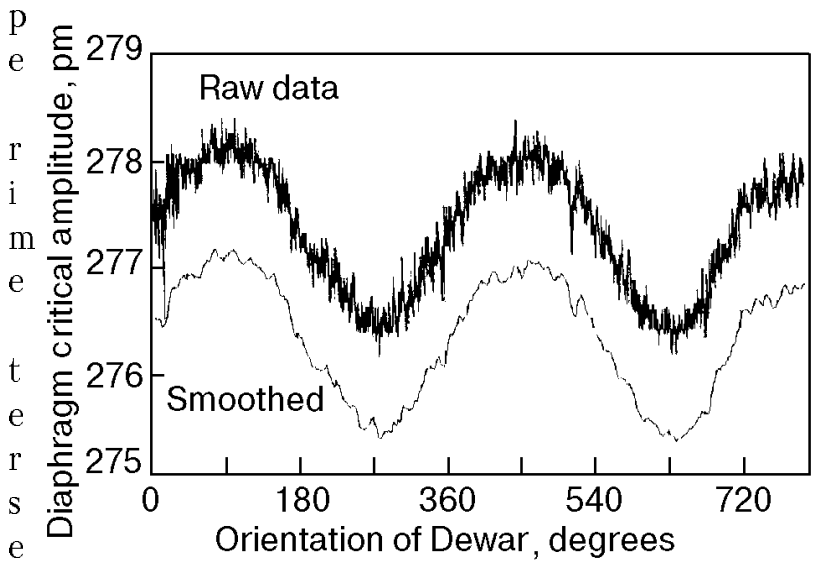

ifig. 1. Modulation of diaphragm critical amplitude from ${ }_{s}$ Earth's rotation. Smoothed curve has been shifted down $0.5 \mathrm{pm}$ for visibility.

i

ng loop with an area of $\approx 1 \cdot 10^{-4} \mathrm{~m}^{2}$. By utilizing silicon processing techniques [9] we are able to integrate the submicron sized aperture with a patterned, thin sensing loop ( $80 \mu \mathrm{m}$ thick $)$. Also, the superfluid flow field is easily calculated in this two dimensional geometry using commercial partial differential equation solving software.

This device shows a staircase response which is characteristic of an rf SQUID. By reorienting the sensing loop with respect to the Earth's rotation vector, we find that the apparent critical velocity in the aperture is modulated as expected, Fig. 1. We drove the superfluid oscillator onto the first step and continuously recorded the apparent critical amplitude while reorienting the Dewar. This reorientation was at a rate of $1 \mathrm{rev} / \mathrm{h}$ and had the effect of changing the Earth's rotational «flux» through the perimeter sensing loop.

The noise in this gyroscope originates from the uncertainty in the superfluid critical velocity at which a vortex is nucleated. Typically, $\Delta v_{c}$ is $\approx 2 \%$ of $v_{c}$. One can show that the rotation resolution in $\mathrm{rad} / \mathrm{s}$ per $\sqrt{\mathrm{Hz}}$ given this uncertainty in critical velocity is

$$
\sqrt{S_{\omega}}=\frac{1+R}{R} \frac{l_{a}}{A_{\mathrm{eff}} \sqrt{8 \pi v_{H}}} \Delta v_{c},
$$

where $R$ is the ratio of the hydrodynamic inductances of the two hole oscillator, $A_{\text {eff }}$ is the effective sensing area, and $v_{H}$ is the oscillator resonance. In our case, $\quad l_{a}=0.25 \cdot 10^{-6} \mathrm{~m}, R=7, \quad A_{\text {eff }}=9.8 \cdot 10^{-5} \mathrm{~m}^{2}$, $v_{H}=66.5 \mathrm{~Hz}, v_{c}=8.4 \mathrm{~m} / \mathrm{s}, \Delta v_{c}=0.22 \mathrm{~m} / \mathrm{s}$, thus $\sqrt{S_{\omega}}=15 \cdot 10^{-6}(\mathrm{rad} / \mathrm{s})(1 / \sqrt{\mathrm{Hz}})$. 


\section{Superfluid gyroscopes and the Lense-Thirring Effect}

It is predicted that the local inertial frame is not the same frame as that attached to the fixed stars. The relative rotation between these two frames is expected to be at the level of tens of milli-arcsec per year, and arises from the general relativistic effect of the rotating Earth on the local inertial frame, the gravitomagnetic field [10]. Motivations for detecting this gravitomagnetic field are found at the most fundamental level of physics. Detection of this effect would provide information to basic questions concerning general relativity and the origins of inertia [11].

Currently, there are two active experiments which intend to measure this new field: LAGEOS [12] and Gravity Probe B (GPB) [13]. The LAGEOS experiments involves the tracking of Earth orbiting satellites. The gravitomagnetic field should precess the plane of the orbit. The GPB experiment uses spinning quartz sphere gyroscopes on an Earth orbiting satellite. In this case, the measurement is of the precession of the gyro axis. Although a superfluid gyroscope is very far from possessing the sensitivity required to detect this effect, it is interesting to compare it to these existing projects.

Previous groups have already suggested the possibility of using superfluid to measure the Lense Thirring field [14,15]. An example of such a scheme is three superfluid gyroscopes oriented mutually perpendicular, attached to a platform carrying a pointing telescope. These gyroscopes provide a measure of absolute rotation about any axis and by combining the output of these gyroscopes with a feedback loop and actuators, one could hold the platform non-rotating with respect to our local inertial frame. Using the telescope to provide a reference to the inertial frame of the distant universe, it is predicted that the observed star would drift as viewed by the telescope at a rate consistent with the Earth's gravitomagnetic field. This experiment is essentially the inverse of the GPB experiment.

In comparison to LAGEOS, both the superfluid gyroscope and GPB are much less sensitive to effects which cause additional rotations, such as the non-spherical mass distribution of the Earth. For LAGEOS, one must model these effects to a precision better that one part in $10^{7}$ to resolve the small residual rotation caused by the general relativistic effect. For GPB, the rotation caused by the Earth's oblateness is about one third the rate of rotation caused by the gravitomagnetic field. A superfluid gyroscope would have a similar low sensitivity to such a non-ideality, and thus would provide a much more direct measurement as compared to the LAGEOS experiment.

As compared to the spinning quartz balls used for GPB, the superfluid gyroscope has a more relaxed constraint on the physical environment: fewer «Near Zero» requirements. The spinning sphere gyroscope requires: exotic fabrication, extreme vacuum, a method to spin-up and suspend the spheres, and ultra-low ambient magnetic and electric field. The superfluid gyroscope does not require such extreme experimental conditions: helium couples very weakly to electromagnetic fields through the small dielectric polarizability; a superfluid gyroscope will never «spin down» since the measured effect is given by a quantum constraint.

The superfluid gyroscope is a rate sensor, while the GPB scheme is an angle sensor. If one used the superfluid gyroscope in a feedback loop as described, the error in the angular orientation will grow with time, $\tau$ : $\sigma_{\theta}=\sqrt{\tau S_{\omega}}$. Thus, the signal to noise ratio will grow only as the square root of time. To obtain a resolution of 1 milli-arcsec after a measurement time of 1 year, a superfluid gyroscope with spectral noise density of $\approx 1 \cdot 10^{-12}(\mathrm{rad} / \mathrm{s})(1 / \sqrt{\mathrm{Hz}})$ is required. This requires an improvement of a factor of $\approx 5 \cdot 10^{6}$ over our demonstrated gyroscope.

As for GPB, the signal from the precessing spinning spheres increases linearly with the measurement time, but only if the spurious torques are negligible. The axis of the spinning sphere is taken to be the reference to the non-rotating inertial frame. If this axis is perturbed the measurement can be lost. With a superfluid gyroscope one is always measuring with respect to the inertial frame, and this frame cannot be «lost» due to some perturbation midway through the measurement.

It is essential to note that we make this comparison to existing experiments with caution. The superfluid gyroscope has only been recently demonstrated with a modest level of sensitivity and it is as yet unclear what problems will be encountered as one attempts to achieve higher sensitivities. Fundamental measurement issues such as low-frequency noise and stability are not yet known.

\section{Conclusions and future directions}

The superfluid gyroscope offers a technique to measure absolute rotation. Given the sensitivity of the first realized devices, it is very interesting to continue to develop increasingly sensitive superfluid gyroscopes. Research efforts to improve the sensitivity of the superfluid gyroscope should be 
focused in two areas as indicated by Eq. (6): increasing the coupling with larger sensing loops, and to find ways to decrease the uncertainty in the critical velocity for vortex nucleation.

The first step to improve rotational coupling would be to wind a much larger area, multi-turn, sensing loop. If we limit the size of the loop to fit into our current apparatus ( $10 \mathrm{~cm}$ dia. Dewar), we could increase the rotational coupling by 1000 in comparison to our current apparatus. Ideally, if one could suppress all forms of non-fundamental noise and disturbance, one could achieve a sensitivity of $5 \cdot 10^{-6}$ of the rotation of the Earth with a measurement time of $1 \mathrm{~h}$. This would be a significant step toward realizing a superfluid gyroscope which could sense the Earth's gravitomagnetic field.

This work is supported by the Office of Naval Research and the National Science Foundation.

1. J. Anandan, Phys. Rev. Lett. 47, 463 (1981).

2. M. Gerdonio and S. Vitale, Phys. Rev. B29, 481 (1984).

3. R.E. Packard and S. Vitale, Phys. Rev. B46, 3540 (1992).
4. O. Avenel and E. Varoquaux, Gzech. J. Phys. 46, 3319 (1996).

5. K. Schwab, N. Bruckner, and R. E. Packard, Nature 386, 585 (1997).

6. K. Schwab, Ph. D. thesis, University of California, Berkeley, USA (1996).

7. J. Anandan, J. Phys. A17, 1367 (1984).

8. B. Cabrera, in: Near Zero: New Frontiers of Physics, J. D. Fairbank, J. B. S. Deaver, C. W. F. Everitt, and P. F. Michelson (eds.), W. H. Freeman and Company, New York (1988), pp. 312-322.

9. K. Schwab, J. Steinhauer, J. Davis, and R. Packard, J. Microelectromechanical Systems 5, 180 (1996).

10. J. Lense and H. Thirring, Phys. Zeits. 29, 156 (1918).

11. I. Cuifolini and J. A. Wheeler, Gravitation and Inertia Princeton University Press, Princeton, New Jersey (1995).

12. D. Smith and P. Dunn, J. Geophys Res. Lett. 7, 437 (1980).

13. C. W. F. Everitt, same as Ref. 8, pp. 587-639.

14. M. Gerdonio, G. A. Prodi, and S. Vitale, General Relativity and Gravitation 20, 83 (1988).

15. B. Cabrera and C. W. F. Everitt, in: Near Zero: New Frontiers of Physics, J. D. Fairbank, J. B. S. Deaver, C. W. F. Everitt, and P. F. Michelson (eds.), W. H. Freeman and Company, New York (1988). 